\title{
A thermodynamically consistent discontinuous Galerkin formulation for interface separation
}

\author{
Daniele Versino $^{\mathrm{a}}$, Hashem M. Mourad ${ }^{\mathrm{a}, *}$, Carlos G. Dávila ${ }^{\mathrm{b}}$, Francis L. Addessio $^{\mathrm{a}}$ \\ ${ }^{a}$ Theoretical Division, T-3, Los Alamos National Laboratory \\ Los Alamos, New Mexico 87545, USA \\ ${ }^{b}$ Structural Mechanics and Concepts Branch, NASA Langley Research Center \\ Hampton, Virginia 23681, USA
}

\begin{abstract}
This paper describes the formulation of an interface damage model, based on the discontinuous Galerkin (DG) method, for the simulation of failure and crack propagation in laminated structures. The DG formulation avoids common difficulties associated with cohesive elements. Specifically, it does not introduce any artificial interfacial compliance and, in explicit dynamic analysis, it leads to a stable time increment size which is unaffected by the presence of stiff massless interfaces. The proposed method is implemented in a finite element setting. Convergence and accuracy are demonstrated in Mode I and mixed-mode delamination in both static and dynamic analyses. Significantly, numerical results obtained using the proposed interface model are found to be independent of the value of the penalty factor that characterizes the DG formulation. By contrast, numerical results obtained using a classical cohesive method are found to be dependent on the cohesive penalty stiffnesses. As a result of this notable advantage, the proposed approach is shown to yield more accurate predictions pertaining to crack propagation under mixed-mode fracture. Furthermore, in explicit dynamic analysis, the stable time increment size calculated with the proposed method is found to be an order of magnitude larger than the maximum allowable value for classical cohesive elements.
\end{abstract}

Keywords: Interface failure, delamination, cohesive-zone models, damage modeling, discontinuous Galerkin method, dynamic structural analysis

\section{Introduction}

Delamination, or interfacial cracking between composite layers, is one of the predominant types of damage in laminated fiber-reinforced composites due to their relatively weak interlaminar strengths. Delamination may arise at discontinuities such as free edges, ply terminations, or transverse cracks caused, for instance, by low velocity impact. The ability to understand and predict delamination failures is essential for ensuring the structural integrity of composite structures.

The simulation of delamination using the finite element method (FEM) is normally performed by means of either the virtual crack closure technique (VCCT) [1], or using cohesive finite elements [2,3]. The VCCT is a linear elastic fracture mechanics-based method that calculates the energy release rate for crack propagation using the assumption that the energy released during delamination propagation equals the work required to close the crack back to its original position. Based on this assumption, the components of the energy release rate are computed from the nodal forces and nodal relative displacements [1]. Delamination growth is predicted when the energy release rate reaches a critical value. There are some difficulties when using the VCCT in the simulation of progressive delamination. The calculation of fracture parameters requires nodal variables and topological information from the nodes ahead and behind the crack front. Such calculations are tedious to perform and may require remeshing for crack propagation. In addition, the VCCT cannot predict the initiation of cracks in undamaged interfaces.

*Corresponding author. Tel: +1 5056062188. Fax: +1 5056655926.

Email address: hmourad@lanl.gov (Hashem M. Mourad) 
The use of cohesive finite elements can overcome some of the difficulties associated with VCCT. Cohesive finite elements can predict both the onset and the non-self-similar propagation of delamination using a traction-separation law that relates crack opening displacements with tractions acting on the interface. Rice's integral [4] ensures that the energy dissipated during the fracture process is equal to the interface fracture energy of the material. However, the simulation of progressive delamination using cohesive elements poses some numerical difficulties, such as those related to the proper definition of the stiffness of the undamaged cohesive layer, the requirement of extremely fine meshes, the convergence difficulties when using implicit analysis methods, and the small time increments required for stability in explicit analyses.

Depending on the form of the traction-separation law, a cohesive zone model (CZM) can be classified as being either intrinsic or extrinsic [5]. Intrinsic cohesive models are characterized by an initial elastic response followed by a softening law that represents crack initiation, nucleation and propagation. Their behavior is that of a spring and the corresponding finite element formulation is based on the penalty method: the internal energy of the cohesive element is a quadratic function of the crack opening displacements multiplied by a penalty stiffness. The penalty stiffness, which accounts for the initial elastic response of the undamaged interface, introduces a small non-physical compliance prior to the onset of fracture. This compliance can affect the wave propagation speed in the undamaged material [6]. In order to overcome the problems caused by this so-called artificial compliance, it is necessary to increase the penalty factor, which imposes severe restrictions on the critical time steps for explicit dynamics, and results in an ill-conditioned tangent matrix when Newton-Raphson solution procedures are used.

Extrinsic cohesive methods have been introduced to address some of the limitations of intrinsic methods: the initial undamaged structure is modeled without cohesive elements, which are introduced only upon satisfaction of a given criterion in a small region of the model $[7,8]$. The extrinsic traction-separation law represents the behavior of the interface outside the elastic region (pure softening response), hence the artificial compliance phenomenon does not manifest itself until the crack begins to propagate. Upon crack closure, the response becomes that of an intrinsic traction-separation law. In order to eliminate the computational effort required to introduce new interfaces, extrinsic cohesive methods have been coupled with discontinuous Galerkin (DG) formulations [5, 9-13]. With this hybrid DG-CZM approach, the interfaces are introduced at the beginning of the analysis in the undamaged structure and the DG formulation overcomes the artificial compliance issue. When a failure criterion is satisfied, the weak form of the equations of motion transitions from that of a DG method to a penalty formulation and an extrinsic traction-separation law is introduced. Reapplying the DG formulation when compression is detected provides a way to eliminate artificial compliance after crack closure [5]. Due to the transition from a DG weak form to a pure penalty formulation, it is necessary to account for the fact that the displacement jump before failure is not exactly zero [11].

When intrinsic or extrinsic cohesive models are employed, it is crucial that the irreversibility of the fracture process be ensured by the interface model. Therefore, potential-based interface models [14] have been developed adopting internal damage variables [15-18]. Nonetheless, most methods relate the damage threshold to a maximum crack opening, a hypothesis which may lead to the violation of the Clausius-Duhem inequality when the crack grows in a varying mode mixity; see Ref. [19].

The subject of the current paper is the development of an interface model based entirely on a DG weak form that overcomes issues encountered with both intrinsic and extrinsic formulations. The DG method used here is an interior penalty method [20-22] and is equipped with an internal damage variable whose evolution is controlled by a potential, written in terms of a function of the traction vector acting on the interface. The damage evolution satisfies the Clausius-Duhem inequality, hence irreversibility is ensured for mixed-mode crack growth. The interface elements are inserted at the element interfaces at the beginning of the analysis and the DG weak form is employed to model the pristine as well as the damaged interface. The energy dissipated during the fracture process is independent of the value of the penalty factor required to stabilize the interior penalty DG formulation. Moreover, the speed of the elastic stress waves does not depend on the penalty factor, and compressive reloading of the interface does not introduce artificial compliance. Additionally, the interfacial contribution to the consistent tangent matrix, used in static or implicit dynamic analysis, lends itself to symmetrization in a straightforward manner.

The damage evolution law employed here produces a linear softening response that relates crack openings and tractions. Although simple, this damage evolution law is capable of representing the two most important physical quantities for crack propagation analysis: interface fracture energies and the interface strengths [23-25]. Moreover, the damage evolution law ensures that the energy dissipated during the varying mode crack growth is equal to the fracture energy obtained from the Benzeggagh-Kenane criterion [26]. 
The remainder of this paper is structured as follows. The equations of motion and their weak form are introduced in the following section. The weak form of the interior penalty DG formulation and its enrichment with a damage variable are presented in Section 3. In Section 4, the thermodynamically consistent framework for the damage evolution is introduced. Subsequently, the interface model is specialized for the analysis of varying mode mixity delamination in Section 5, and the computational formulation is presented in Section 6. In Section 7, some characteristics of the present interface model are discussed. Several numerical simulations involving laminated and homogeneous plates are carried out in Section 8 to assess the performance of the present formulation in the contexts of static and dynamic analysis. Finally, a summary and concluding remarks are presented in Section 9.

\section{Weak form of the equations of motion}

We consider a n-dimensional domain, $\Omega$, defined in a Cartesian coordinate system. The boundary, $\Gamma$, of the domain is split into two disjoint subsets, $\Gamma_{u}$ and $\Gamma_{\sigma}$, where essential and natural boundary conditions, respectively, are imposed. The principle of conservation of linear momentum under the small deformation assumption can hence be stated as follows

$$
\begin{aligned}
\nabla \cdot \boldsymbol{\sigma}+\boldsymbol{b} & =\rho \ddot{\boldsymbol{u}} & & \text { in } \Omega, \\
\boldsymbol{\sigma} \cdot \boldsymbol{n} & =\overline{\boldsymbol{h}} & & \text { on } \Gamma_{\sigma}, \\
\boldsymbol{u} & =\overline{\boldsymbol{u}} & & \text { on } \Gamma_{u},
\end{aligned}
$$

for all time $\tau$. Here, $\boldsymbol{u}$ denotes the displacement, $\rho$ is the mass density, $\boldsymbol{b}$ is the body force per unit volume, $\boldsymbol{\sigma}$ is the Cauchy stress tensor, $\overline{\boldsymbol{u}}$ and $\overline{\boldsymbol{h}}$ are the specified boundary displacements and tractions, respectively, and $\boldsymbol{n}$ is the outward unit normal to $\Gamma_{\sigma}$. Initial conditions are given by

$$
\begin{array}{ll}
\boldsymbol{u}=\boldsymbol{u}_{0} & \text { in } \Omega, \text { at } \tau=0, \\
\dot{\boldsymbol{u}}=\dot{\boldsymbol{u}}_{0} & \text { in } \Omega, \text { at } \tau=0,
\end{array}
$$

where $\boldsymbol{u}_{0}$ and $\dot{\boldsymbol{u}}_{0}$ are known. The domain is approximated by a discretization $\Omega^{h} \approx \Omega$ of non-overlapping finite elements belonging to the set $\Phi_{\Omega^{e}}$ such that

$$
\Omega^{h}=\bigcup_{\Omega^{e} \in \Phi_{\Omega^{e}}} \Omega^{e}
$$

where $\Omega^{e}$ denotes an individual element. A set comprising the edges of the elements, $\Phi_{\Gamma^{e}}$, is defined

$$
\Phi_{\Gamma^{e}}=\Phi_{\Gamma_{I}^{e}} \cup \Phi_{\Gamma_{u}^{e}} \cup \Phi_{\Gamma_{\sigma}^{e}}
$$

where $\Phi_{\Gamma_{u}^{e}}, \Phi_{\Gamma_{\sigma}^{e}}$ and $\Phi_{\Gamma_{I}^{e}}$ respectively represent the set of edges with specified boundary displacements, the set of edges with assigned boundary tractions and the set of edges that do not lie on the domain's boundary (interior edges). Moreover, the following properties hold $\Phi_{\Gamma_{I}^{e}} \cap \Phi_{\Gamma_{u}^{e}}=\emptyset, \Phi_{\Gamma_{I}^{e}} \cap \Phi_{\Gamma_{\sigma}^{e}}=\emptyset$ and $\Phi_{\Gamma_{u}^{e}} \cap \Phi_{\Gamma_{\sigma}^{e}}=\emptyset$.

Multiplying Eqs. (1)-(2) by a weighting function, $\boldsymbol{w}$, integrating the product over the domain, and making use of the divergence theorem, results in the weak form of the equations of motion, which can be written as follows:

$$
\sum_{\Omega^{e} \in \Phi_{\Omega^{e}}}\left[\Pi_{\sigma}^{\Omega}(\boldsymbol{w}, \boldsymbol{u})+\Pi_{\rho}^{\Omega}(\boldsymbol{w}, \boldsymbol{u})\right]-\sum_{\Gamma_{I}^{e} \in \Phi_{\Gamma_{I}^{e}}} \Pi_{I}^{\Gamma}(\boldsymbol{w}, \boldsymbol{u})-\sum_{\Gamma_{\sigma}^{e} \in \Phi_{\Gamma_{\sigma}^{e}}} \Pi_{\sigma}^{\Gamma}(\boldsymbol{w}, \boldsymbol{u})=0
$$

where

$$
\Pi_{\sigma}^{\Omega}=\int_{\Omega^{e}}[\boldsymbol{\varepsilon}(\boldsymbol{w})]: \sigma \mathrm{d} \Omega^{e},
$$

is the internal work associated with the deformation of the element in the presence of stress, and where the strain $\boldsymbol{\varepsilon}(\boldsymbol{u})=\frac{1}{2}\left(\nabla \boldsymbol{u}+\nabla^{T} \boldsymbol{u}\right)$ is defined as the symmetric gradient of the displacement $\boldsymbol{u}$. The inertial contribution is given by

$$
\Pi_{\rho}^{\Omega}=\int_{\Omega^{e}} \rho \boldsymbol{w} \cdot \ddot{\boldsymbol{u}} \mathrm{d} \Omega^{e},
$$


and the third term in Eq. (8) represents the virtual wok due to the tractions acting on an interior interface shared among two adjacent elements, $\Omega^{e^{-}}$and $\Omega^{e^{+}}$:

$$
\Pi_{I}^{\Gamma}=\int_{\Gamma_{I}^{e}}\left(\boldsymbol{w}^{-} \cdot \boldsymbol{\sigma}^{-} \cdot \boldsymbol{n}^{-}+\boldsymbol{w}^{+} \cdot \boldsymbol{\sigma}^{+} \cdot \boldsymbol{n}^{+}\right) \mathrm{d} \Gamma_{I}^{e},
$$

where the notation $\bullet^{-}\left(\bullet^{+}\right)$indicates the value assumed by the quantity $\bullet$, defined on the element $\Omega^{e^{-}}\left(\Omega^{e^{+}}\right)$, on the interface shared by $\Omega^{e^{-}}$and $\Omega^{e^{+}}$. The virtual work term arising from the prescribed Neumann conditions is

$$
\Pi_{\sigma}^{\Gamma}=\int_{\Gamma_{\sigma}^{e}}(\boldsymbol{w} \cdot \overline{\boldsymbol{h}}) \mathrm{d} \Gamma_{\sigma}^{e} .
$$

The remainder of the paper is concerned with the treatment of the $\Pi_{I}^{\Gamma}$ term during the interface separation process.

\section{Discontinuous Galerkin formulation for cohesive interfaces}

In order to introduce the DG fluxes into the $\Pi_{I}^{\Gamma}$ term, it is useful to define the jump, $\llbracket \bullet \rrbracket$, and average, $\langle\bullet\rangle$, operators acting on the quantity $\bullet$, such that

$$
\begin{aligned}
\llbracket \bullet \rrbracket & =\left[\bullet^{-}-\bullet^{+}\right], \\
\langle\bullet\rangle & =\frac{1}{2}\left[\bullet^{-}+\bullet^{+}\right] .
\end{aligned}
$$

Moreover, the normal to the interface is denoted by $\boldsymbol{n}$, with $\boldsymbol{n}=\boldsymbol{n}^{-}=-\boldsymbol{n}^{+}$. After the introduction of the so-called interior penalty DG fluxes [27] in Eq. (11), the $\Pi_{I}^{\Gamma}$ term can be written as

$$
\Pi_{I}^{\Gamma}(\boldsymbol{w}, \boldsymbol{u})=\int_{\Gamma_{I}^{e}}(\llbracket \boldsymbol{w} \rrbracket \cdot\langle\boldsymbol{\sigma}(\boldsymbol{u}) \cdot \boldsymbol{n}\rangle+\langle\boldsymbol{\sigma}(\boldsymbol{w}) \cdot \boldsymbol{n}\rangle \cdot \llbracket \boldsymbol{u} \rrbracket-\llbracket \boldsymbol{w} \rrbracket \cdot \boldsymbol{\alpha} \cdot \llbracket \boldsymbol{u} \rrbracket) \mathrm{d} \Gamma_{I}^{e},
$$

with

$$
\boldsymbol{\alpha}=\frac{\lambda}{h_{s}}\langle\boldsymbol{n} \cdot \mathbb{C} \cdot \boldsymbol{n}\rangle,
$$

where $h_{s}$ is a characteristic length, $\mathbb{C}$ is the material's tensor of tangent moduli and $\lambda$ an arbitrary scalar penalty parameter [10]. It should be remarked that the second and the third terms in Eq. (15) are respectively responsible for the symmetrization of the method and for its stabilization. In order to simplify the formulation, it is common practice to neglect the second term in Eq. (15) and obtain a non-symmetric weak form [10]. Therefore, the contribution of the interior interfaces to the virtual work becomes

$$
\Pi_{I}^{\Gamma}(\boldsymbol{w}, \boldsymbol{u})=\int_{\Gamma_{I}^{e}}(\llbracket \boldsymbol{w} \rrbracket \cdot\langle\boldsymbol{\sigma} \cdot \boldsymbol{n}\rangle-\llbracket \boldsymbol{w} \rrbracket \cdot \boldsymbol{\alpha} \cdot \llbracket \boldsymbol{u} \rrbracket) \mathrm{d} \Gamma_{I}^{e},
$$

or

$$
\Pi_{I}^{\Gamma}(\boldsymbol{w}, \boldsymbol{u})=\int_{\Gamma_{I}^{e}} \llbracket \boldsymbol{w} \rrbracket \cdot \boldsymbol{t}^{*} \mathrm{~d} \Gamma_{I}^{e},
$$

where

$$
\boldsymbol{t}^{*}(\boldsymbol{u})=\langle\boldsymbol{\sigma} \cdot \boldsymbol{n}\rangle-\boldsymbol{\alpha} \cdot \llbracket \boldsymbol{u} \rrbracket .
$$

The first term and the second term on the right-hand side of Eq. (19) respectively represent the contribution of the DG fluxes and the penalty term necessary to stabilize the DG method. It is possible to recover a penalty formulation if $\langle\boldsymbol{\sigma} \cdot \boldsymbol{n}\rangle \equiv 0$. 
The hybrid DG-CZM formulation has been proposed for modeling delamination [5, 9]. The DG formulation, Eq. (18), is used before crack initiation, while an extrinsic traction-separation law, $\boldsymbol{t}^{s}$, is introduced upon satisfaction of a given failure criterion. In the context of such a formulation, the interfacial virtual work contribution can be written [9] as:

$$
\Pi_{I}^{\Gamma}(\boldsymbol{w}, \boldsymbol{u})=\int_{\Gamma_{I}^{e}} \llbracket \boldsymbol{w} \rrbracket \cdot t \mathrm{~d} \Gamma_{I}^{e}
$$

with

$$
\boldsymbol{t}(\boldsymbol{u})=(1-a) \boldsymbol{t}^{*}(\boldsymbol{u})+a \boldsymbol{t}^{s}(\llbracket \boldsymbol{u} \rrbracket)
$$

where $\boldsymbol{t}^{s}$ is a function of the displacement jump and of the interface parameters such as fracture energy and the interface strength. The binary parameter $a$ is zero when the interface is undamaged and assumes a unit value when the failure criterion is met. In order to guarantee irreversibility, the value of $a$ cannot decrease and hence the tractionseparation law represents both loading and unloading processes. The underlying assumption is that the displacement jump, $\llbracket \boldsymbol{u} \rrbracket$, is equivalent to the crack opening and that $\llbracket \boldsymbol{u} \rrbracket \equiv \mathbf{0}$ before crack initiation. Due to the DG formulation, however, displacement continuity before crack initiation is only enforced weakly and hence $0 \leq\|\llbracket \boldsymbol{u} \rrbracket\| \leq \epsilon$ with $\epsilon \ll 1$. A possible remedy to this problem is to define the cohesive traction separation law as a function of a new crack opening $\llbracket \boldsymbol{u} \rrbracket^{*}$ given by $[11]$ :

$$
\llbracket \boldsymbol{u} \rrbracket^{*}=\llbracket \boldsymbol{u} \rrbracket-\llbracket \boldsymbol{u} \rrbracket_{\tau=\tau^{c}},
$$

where $\llbracket \boldsymbol{u} \rrbracket_{\tau=\tau^{c}}$ is the displacement jump at crack initiation (time $\tau=\tau_{c}$ ).

In the current paper, a different approach is proposed: the DG formulation is used to model the interface behavior regardless of its undamaged/damaged state and no traction-separation law is employed. The binary variable $a$ is replaced by a damage variable $D$ varying continuously in the range $[0,1]$. The DG fluxes are considered to be acting only on the undamaged portion of the interface and Eq. (21) is replaced with

$$
\boldsymbol{t}(\boldsymbol{u})=(1-D) \boldsymbol{t}^{*}(\boldsymbol{u})
$$

The virtual work contribution of the interface becomes

$$
\Pi_{I}^{\Gamma}(\boldsymbol{w}, \boldsymbol{u}, D)=\int_{\Gamma_{I}^{e}} \llbracket \boldsymbol{w} \rrbracket \cdot t^{*}(1-D) \mathrm{d} \Gamma_{I}^{e},
$$

where $(1-D) \mathrm{d} \Gamma_{I}^{e}$ can be regarded as the undamaged area of the interface. In order to avoid interpenetration along the damaged portion of the interface, $D \mathrm{~d} \Gamma_{I}^{e}$, an additional term is added to Eq. (24) leading to the following expression for the virtual work of the interface

$$
\Pi_{I}^{\Gamma}(\boldsymbol{w}, \boldsymbol{u}, D)=\int_{\Gamma_{I}^{e}} \llbracket \boldsymbol{w} \rrbracket \cdot \boldsymbol{t}^{*}(1-D) \mathrm{d} \Gamma_{I}^{e}+\int_{\Gamma_{I}^{e}} \llbracket \boldsymbol{w} \rrbracket \cdot \boldsymbol{n}\left\{-\boldsymbol{t}^{*} \cdot \boldsymbol{n}\right\} D \mathrm{~d} \Gamma_{I}^{e},
$$

where $\{\bullet\}$ signify the Macaulay brackets, defined as $\{\bullet\}=\frac{1}{2}(|\bullet|+\bullet)$. To complete the formulation it is now necessary to define a thermodynamically consistent evolution law for the damage variable $D$.

\section{Damage model}

A free energy potential, $\Psi$, is assumed to exist and to depend on the numerical fluxes acting across the interface, $\boldsymbol{t}^{*}$, and on the damage variable, $D$ :

$$
\Psi\left(t^{*}, D\right)=(1-D) \Psi^{0}\left(t^{*}\right)
$$

where $\Psi^{0}$ is the initial elastic stored energy function of the undamaged interface such that [28]:

$$
\Psi^{0}(\mathbf{0})=0,
$$

$$
\left.\frac{\partial \Psi^{0}}{\partial t^{*}}\right|_{t^{*}=\mathbf{0}}=0
$$


The thermodynamic force conjugate to the damage variable is defined as

$$
Y=-\frac{\partial \Psi}{\partial D}=\Psi^{0}
$$

and the thermodynamic variable conjugate to $t^{*}$ is given by

$$
\boldsymbol{\delta}=\frac{\partial \Psi}{\partial \boldsymbol{t}^{*}}=(1-D) \frac{\partial \Psi^{0}}{\partial \boldsymbol{t}^{*}}
$$

Therefore, $\delta$ may be regarded as the elastic part of the crack opening and it should be noted that even in the elastic regime (before crack initiation) $\boldsymbol{\delta}$ is in general not equal to $\llbracket \boldsymbol{u} \rrbracket$. The Clausius-Duhem inequality, in the isothermal case, takes the form

$$
-\dot{\Psi}+\boldsymbol{\delta} \cdot \dot{\boldsymbol{t}}^{*} \geq 0
$$

which, considering that

$$
\dot{\Psi}=\frac{\partial \Psi}{\partial t^{*}} \cdot \dot{\boldsymbol{t}}^{*}+\frac{\partial \Psi}{\partial D} \dot{D}
$$

may be simplified to

$$
Y \dot{D} \geq 0
$$

It is clear that having $Y \geq 0$ implies that $\dot{D} \geq 0$ which prevents the damaged interface from healing. In accordance with the conditions (27), the initial free energy potential is assumed to be a quadratic form of the interface tractions and to have the simple form

$$
\Psi^{0}\left(t^{*}\right)=\frac{1}{2} t^{*} \cdot \mathbf{C}^{e} \cdot \boldsymbol{t}^{*}
$$

with

$$
\mathbf{C}^{e}=\left(\mathbf{D}^{e}\right)^{-1},
$$

where $\mathbf{D}^{e}$ is a rank-two tensor characterizing the elastic stiffness of the interface. Therefore the thermodynamic variable conjugate to $\boldsymbol{t}^{*}$ becomes

$$
\boldsymbol{\delta}=(1-D) \mathbf{C}^{e} \cdot \boldsymbol{t}^{*}
$$

In order to completely define the damage state of an interface we introduce the damage surface, $f=0$, enclosing all admissible values of $Y$ :

$$
f(Y, r):=Y-r \leq 0,
$$

and the evolution of the damage variable $D$ takes the form

$$
\begin{aligned}
\dot{D} & =\dot{\mu} h(Y, D), \\
\dot{r} & =\dot{\mu},
\end{aligned}
$$

where $h$ controls the evolution of the damage (see Section 5) and where $\dot{\mu} \geq 0$ is a damage consistency parameter that defines damage loading/unloading conditions according to the Kuhn-Tucker relations [28]:

$$
\dot{\mu} \geq 0, \quad f \leq 0, \quad \dot{\mu} f=0 .
$$

When iterative solvers based on Newton-Raphson are employed, it is necessary to compute the consistent tangent matrix of the interface model, $\mathbf{D}$, that relates the rates of $\boldsymbol{t}$ and $\boldsymbol{t}^{*}$ according to:

$$
\dot{\boldsymbol{t}}=\mathbf{D} \cdot \dot{\boldsymbol{t}}^{*} .
$$


In order to compute the consistent tangent for the interface model it is convenient to write the rate form of Eq. (23)

$$
\dot{\boldsymbol{t}}=(1-D) \dot{\boldsymbol{t}}^{*}-\boldsymbol{t}^{*} \dot{D}
$$

and to differentiate Eq. (36) with respect to $\tau$

$$
\dot{f}=\dot{Y}-\dot{r}=0
$$

When $\dot{D}>0$ Eq. (41) together with (28), (33), (37)-(38) and (42) gives

$$
\dot{\boldsymbol{t}}=\left[(1-D) \mathbf{1}-h \boldsymbol{t}^{*}\left(\mathbf{C}^{e} \cdot \boldsymbol{t}^{*}\right)\right] \cdot \dot{\boldsymbol{t}}^{*}
$$

where 1 is the rank-two identity tensor. Comparing Eq. (40) and Eq. (43) leads to the following expression for the consistent tangent matrix:

$$
\mathbf{D}=(1-D) \mathbf{1}-h \boldsymbol{t}^{*}\left(\mathbf{C}^{e} \cdot \boldsymbol{t}^{*}\right)
$$

It is worth noting that $\mathbf{D}$ is always symmetric if $\mathbf{D}^{e}$ is diagonal. Nonetheless, in the general case, $\mathbf{D}$ should be regarded as non-symmetric. Clearly, when $\dot{D}=0$, the interfacial consistent tangent matrix is given by

$$
\mathbf{D}=(1-D) \mathbf{1}
$$

\section{Definition of the interface parameters}

The formulation introduced in the preceding sections is specialized for a two-dimensional interface subject to Mode I (normal) and Mode II (shear or sliding) delaminations. At a given time $\tau=\bar{\tau}$, the energy dissipated during a delamination process is given as in [17] by

$$
\mathrm{G}=\int_{0}^{\bar{\tau}} Y \dot{D} \mathrm{~d} \tau .
$$

The dissipated energy is limited by the interface fracture energy defined as

$$
\mathrm{G}^{c}=\int_{0}^{\infty} Y \dot{D} \mathrm{~d} \tau
$$

i.e. $\mathrm{G} \leq \mathrm{G}^{c}$.

Fracture energies for Mode I, $\mathrm{G}_{\mathrm{I}}^{c}$, and Mode II, $\mathrm{G}_{\mathrm{II}}^{c}$ can be measured experimentally. From the Mode I and Mode II fracture energies, it is possible to compute the fracture energy, $\mathrm{G}^{c}$, corresponding to a given load mixity employing some criterion. Among the several criteria available in literature (see Refs. [15, 29]), the Benzeggagh and Kenane (B-K) criterion [26] is employed throughout this work. The B-K criterion takes the following form

$$
\mathrm{G}_{\mathrm{I}}^{c}+\left(\mathrm{G}_{\mathrm{II}}^{c}-\mathrm{G}_{\mathrm{I}}^{c}\right)\left(\frac{\mathrm{G}_{\mathrm{II}}}{\mathrm{G}_{\mathrm{I}}+\mathrm{G}_{\mathrm{II}}}\right)^{\eta}=\mathrm{G}^{c},
$$

where $\frac{\mathrm{G}_{\mathrm{II}}}{\mathrm{G}_{\mathrm{I}}+\mathrm{G}_{\mathrm{II}}}$ is the mode mixity ratio, $\mathrm{B}$, given in terms of crack opening $[18,30]$ :

$$
\mathrm{B}=\frac{\left|\delta_{1}\right|^{2}}{\left|\delta_{1}\right|^{2}+\left|\delta_{2}\right|^{2}},
$$

with $\delta_{1}$ and $\delta_{2}$ being the components of the crack opening displacements $\delta$. Therefore, the fracture energy for a mixed-load delamination, in accordance with the B-K criterion, can be written as

$$
\mathrm{G}_{\mathrm{I}}^{c}+\left(\mathrm{G}_{\mathrm{II}}^{c}-\mathrm{G}_{\mathrm{I}}^{c}\right) \mathrm{B}^{\eta}=\mathrm{G}^{c}
$$


In order to define the damage evolution it is necessary to determine the functions $h$ and $r$ which depend on the damage variable, on the fracture energies and on the interfacial strengths. Once $h$ or $r$ is defined, it is possible to derive the other function through Eq. (37) and Eq. (38). It is assumed herein that the function $r$ is given by a power law

$$
r=\frac{Y^{c}}{(1-H D)^{2}}
$$

such that $r_{(D=0)}=Y^{c}$ where

$$
Y^{c}=\frac{1}{2} \boldsymbol{t}^{c} \cdot \mathbf{C}^{e} \cdot \boldsymbol{t}^{c}
$$

with $\boldsymbol{t}^{c}=\left[t_{\mathrm{II}}^{c}, t_{\mathrm{I}}^{c}\right]^{T}$. The not yet determined scalar parameter $H$ is introduced to ensure that the total energy dissipated during the delamination process equals the interface fracture energy for the current mode mixity. Moreover, the assumed expression for $r$ yields to a linear softening relation between $\llbracket \boldsymbol{u} \rrbracket$ and $\boldsymbol{t}$ for pure Mode I and pure Mode II delaminations, see Fig. $3 \mathrm{a}$ in Section 8. Taking the time derivative of $r$ obtains

$$
\dot{r}=\frac{2 H Y^{c}}{(1-H D)^{3}} \dot{D}=\frac{1}{h} \dot{D},
$$

and substituting Eq. (53) into Eq. (37) and Eq. (38) can determine the $h$ function, corresponding to the assumed $r$, as

$$
h=\frac{1}{2 H Y^{c}}(1-H D)^{3} .
$$

The damage envelope for the particular choice of $r$ is obtained after substitution of Eq. (51) into Eq. (36)

$$
f(Y, D):=Y-\frac{Y^{c}}{(1-H D)^{2}} \leq 0
$$

and considering that $\dot{D}>0$ implies $f=0$ yields

$$
\mathrm{G}^{c}=\int_{0}^{\infty} Y \dot{D} \mathrm{~d} \tau=\int_{0}^{1} Y \mathrm{~d} D=\int_{0}^{1} \frac{Y^{c}}{(1-H D)^{2}} \mathrm{~d} D,
$$

which, after manipulation provides

$$
H=1-\frac{Y^{c}}{\mathrm{G}^{c}}
$$

It is worth remarking that Eq. (57) is responsible for enforcing the condition in Eq. (47) and that $r_{(D=1)}=\frac{\left(\mathrm{G}^{c}\right)^{2}}{Y^{c}}>0$.

When the current state is outside the damage surface, $f>0$, it is possible to compute the new value of the damage variable for which $f=0$ directly from Eq. (55)

$$
D=\frac{1}{H}\left(\sqrt{\frac{Y^{c}}{Y}}-1\right),
$$

without using an iterative solver.

\section{Finite element implementation}

Bilinear shape functions, $\mathbf{N}$, are used to approximate the two-dimensional displacements and associated weighting functions

$$
\boldsymbol{u}=\mathbf{N} \mathbf{d}_{u}, \quad \boldsymbol{w}=\mathbf{N} \mathbf{d}_{w},
$$


where $\mathbf{d}_{u}$ and $\mathbf{d}_{w}$ are the vectors of nodal values. Moreover, the elements and the interfaces are assumed to be in plane stress state. The element level residual vector, $\mathbf{r}_{\sigma}^{\Omega}$, and consistent tangent matrix, $\mathbf{K}_{\sigma}^{\Omega}$, are derived through linearization of the $\Pi_{\sigma}^{\Omega}$ term in (8):

$$
\mathbf{r}_{\sigma}^{\Omega}=\frac{\partial \Pi_{\sigma}^{\Omega}}{\partial \mathbf{d}_{w}}, \quad \quad \mathbf{K}_{\sigma}^{\Omega}=\frac{\partial \mathbf{r}_{\sigma}^{\Omega}}{\partial \mathbf{d}_{u}}
$$

The standard procedure [31] is employed to obtain the element level mass matrix from $\Pi_{\rho}^{\Omega}$.

Linearization of the $\Pi_{I}^{\Gamma}$ term in Eq. (25) leads to the following definitions of the interface level residual vector, $\mathbf{r}_{I}^{\Gamma}$, and tangent matrix, $\mathbf{K}_{I}^{\Gamma}$ :

$$
\mathbf{r}_{I}^{\Gamma}=\frac{\partial \Pi_{I}^{\Gamma}}{\partial \mathbf{d}_{w}^{\Gamma}}, \quad \quad \mathbf{K}_{I}^{\Gamma}=\frac{\partial \mathbf{r}_{I}^{\Gamma}}{\partial \mathbf{d}_{u}^{\Gamma}}
$$

with

$$
\mathbf{d}_{u}^{\Gamma}=\left[\mathbf{d}_{u}^{-}, \mathbf{d}_{u}^{+}\right], \quad \mathbf{d}_{w}^{\Gamma}=\left[\mathbf{d}_{w}^{-}, \mathbf{d}_{w}^{+}\right],
$$

where $\mathbf{d}_{u}^{-}$and $\mathbf{d}_{u}^{+}$denote the vectors of nodal variables of the element $\Omega^{e^{-}}$and $\Omega^{e^{+}}$respectively. Derivation of the interface residual is straightforward

$$
\mathbf{r}_{I}^{\Gamma}=\frac{\partial \Pi_{I}^{\Gamma}}{\partial \mathbf{d}_{w}^{\Gamma}}=\frac{\partial \Pi_{I}^{\Gamma}}{\partial \llbracket \boldsymbol{w} \rrbracket} \cdot \frac{\partial \llbracket \boldsymbol{w} \rrbracket}{\partial \mathbf{d}_{w}^{\Gamma}}
$$

whereas computation of the interface level tangent matrix requires application of the chain rule:

$$
\frac{\partial \boldsymbol{t}}{\partial \mathbf{d}_{u}^{\Gamma}}=\frac{\partial \boldsymbol{t}}{\partial \boldsymbol{t}^{*}} \cdot \frac{\partial \boldsymbol{t}^{*}}{\partial \mathbf{d}_{u}^{\Gamma}}=\frac{\partial \dot{\boldsymbol{t}}}{\partial \dot{\boldsymbol{t}}^{*}} \cdot \frac{\partial \boldsymbol{t}^{*}}{\partial \mathbf{d}_{u}^{\Gamma}}=\mathbf{D} \cdot\left(\frac{\partial}{\partial \mathbf{d}_{u}^{\Gamma}}\langle\boldsymbol{\sigma} \cdot \boldsymbol{n}\rangle-\boldsymbol{\alpha} \cdot \frac{\partial \llbracket \boldsymbol{u} \rrbracket}{\partial \mathbf{d}_{u}^{\Gamma}}\right),
$$

where $\mathbf{D}$ is given in Eqs. (44) and (45). A two-point Gauss-Legendre quadrature rule is used to compute the integral in Eq. (25) and stresses at the quadrature points on the interface are computed directly from the shape functions.

As observed in Section 3, the interface level tangent matrix $\mathbf{K}_{I}^{\Gamma}$ is non-symmetric. When implicit time integration schemes are employed, it is convenient to use a symmetric tangent matrix, $\mathbf{K}_{I}^{\Gamma S}$, which is obtained through symmetrization of $\mathbf{K}_{I}^{\Gamma}$

$$
\mathbf{K}_{I}^{\Gamma S}=\frac{1}{2}\left(\mathbf{K}_{I}^{\Gamma}+\left(\mathbf{K}_{I}^{\Gamma}\right)^{T}\right)
$$

In Section 4, the elastic response of the interface has been characterized by the rank-two tensor, $\mathbf{D}^{e}$, that is assumed to be given by

$$
\mathbf{D}^{e}=\boldsymbol{\alpha}=\frac{\lambda}{h_{s}}\langle\boldsymbol{n} \cdot \mathbb{C} \cdot \boldsymbol{n}\rangle
$$

It should be noted that this choice of $\mathbf{D}^{e}$ is arbitrary and several other options are possible. Nonetheless, choosing the form in Eq. (66) provides, upon simplification of the DG formulation to a penalty method, the classical penalty-based formulation employed in Refs. [17, 18, 32], among others. The penalty form is recovered when the first term in Eq. (19) is neglected leading to

$$
\boldsymbol{t}^{*}(\boldsymbol{u})=-\boldsymbol{\alpha} \cdot \llbracket \boldsymbol{u} \rrbracket
$$

and, due to Eq. (66), when the response of the interface is elastic (i.e. undamaged interface), the following property holds $\llbracket \boldsymbol{u} \rrbracket=\boldsymbol{\delta}$.

The interface model formulated in this paper has been implemented in ABAQUS (Standard and Explicit) [33] as a user element. As shown in Fig. 1, this finite element has eight nodes, four for $\Omega^{e^{-}}$and four for $\Omega^{e^{+}}$, to allow for computing the traction at the interface. The solution procedure for the interface model has been summarized in Algorithm 1. 


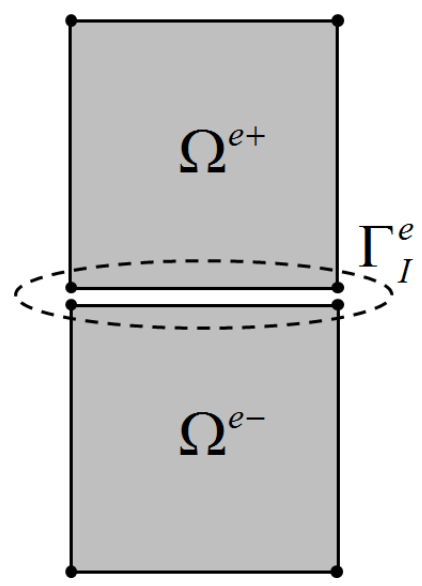

Figure 1: Interface element configuration

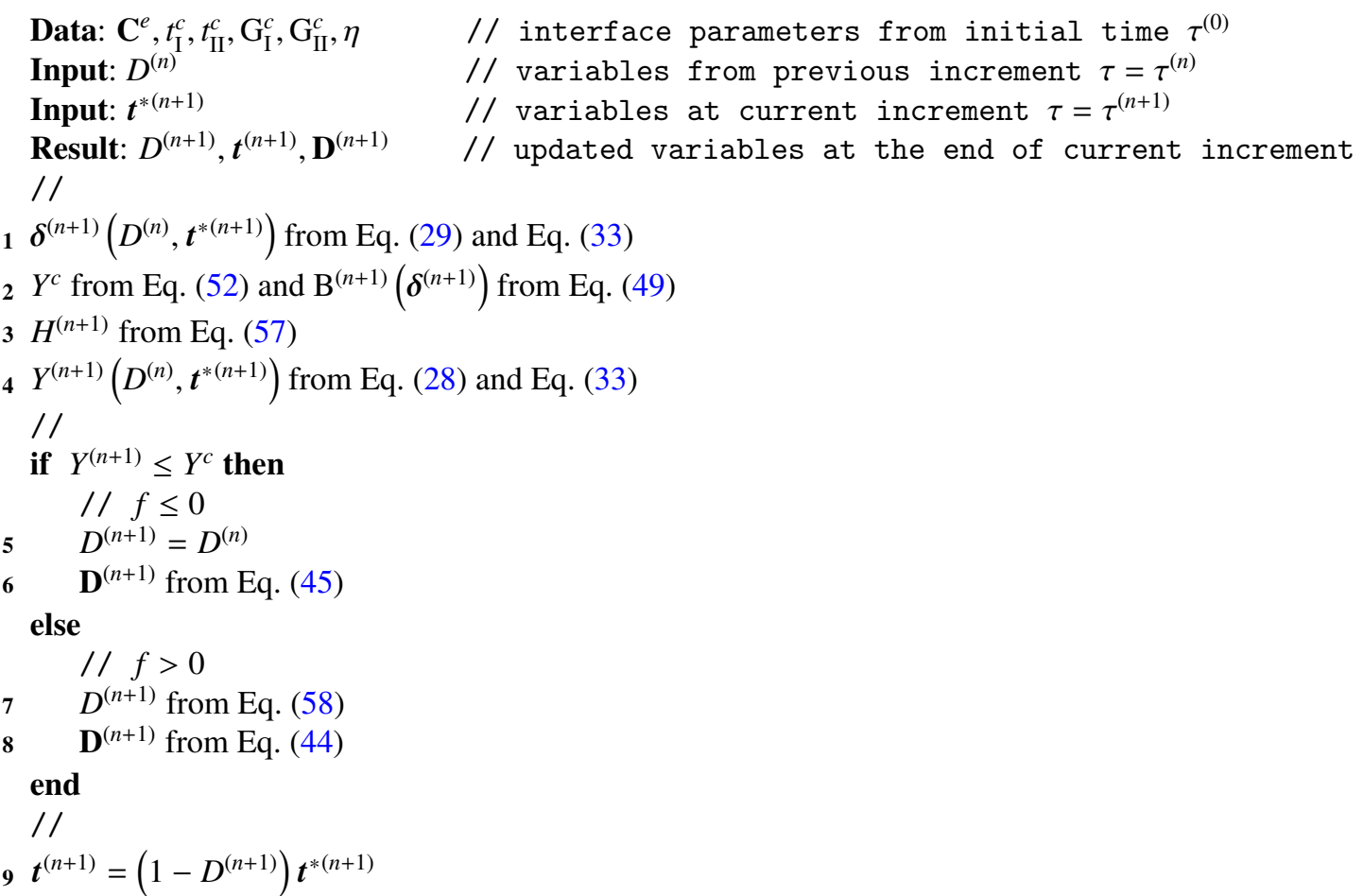

Algorithm 1: Summary of the interface model 


\section{Additional considerations}

In Section 5 the damage model has been specialized for a two dimensional interface and $r$ has been assumed to have the form in Eq. (51) which leads to

$$
r_{(D=0)}=Y^{c}, \quad r_{(D=1)}=\frac{\left(\mathrm{G}^{c}\right)^{2}}{Y^{c}} .
$$

The evolution equation (38) for $r$ requires that $\dot{r} \geq 0$ and hence $r_{(D=1)} \geq r_{(D=0)}$ which yields

$$
G^{c} \geq Y^{c} .
$$

The implications of Eq. (69) are investigated through the simple one-dimensional problem studied in [34]. A clamped bar of total length $L$ is subjected to uniform displacement at the free end. The bar is made of an isotropic material with Young's modulus $E$ and null Poisson's ratio and an interface with fracture energy $\mathrm{G}^{c}$ and strength $t^{c}$ is assumed to exist. From Eq. (66) the interface stiffness is

$$
\mathbf{D}^{e}=\frac{\lambda}{h_{s}} E
$$

and $Y c$ from Eq. (52) becomes

$$
Y^{c}=\frac{1}{2} \frac{h_{s}}{\lambda E}\left(t^{c}\right)^{2},
$$

which substituted into Eq. (69) leads to

$$
\frac{h_{s}}{\lambda} \leq 2 \frac{\mathrm{G}^{c} E}{\left(t^{c}\right)^{2}} .
$$

The condition for the stability of the response of the bar, under displacement control and after damage initiation, is derived in [34] as

$$
L \leq 2 \frac{\mathrm{G}^{c} E}{\left(t^{c}\right)^{2}}
$$

which implies $h_{s}=\lambda L$. As observed in [34], the right-hand side of Eq. (72) is "a characteristic dimension associated with material properties that is typically much smaller than the structural dimensions" and hence the shape of the softening response does not affect the damage propagation process.

Although crack growth is governed by linear elastic fracture mechanics (LFEM), the delamination propagation in classical cohesive elements is influenced by the $\frac{t_{I}^{c}}{t_{\Pi}^{c}}$ ratio $[30,35]$. In [30] it is demonstrated that $\frac{t_{c}^{c}}{t_{\Pi}^{c}}$ cannot vary freely and that the following constraint

$$
t_{\mathrm{II}}^{c}=t_{\mathrm{I}}^{c} \sqrt{\frac{\mathrm{G}_{\mathrm{II}}^{c}}{\mathrm{G}_{\mathrm{I}}^{c}}},
$$

must hold in order to ensure $\dot{D} \geq 0$. The assumption underling the derivation of the present interface model (Section 4) is that $r$ is function of $Y$ and $D$ only. This yields

$$
\frac{\partial r}{\partial \mathrm{B}} \dot{\mathrm{B}} \equiv 0,
$$

which is satisfied by $\dot{\mathrm{B}} \equiv 0$. Therefore, assuming that the mode mixity does not change from $\tau^{(n)}$ to $\tau^{(n+1)}$ permits to ignore condition (74). It is possible to assume that $r=r(Y, D, \mathrm{~B})$ but this is beyond the current scope of the paper. 


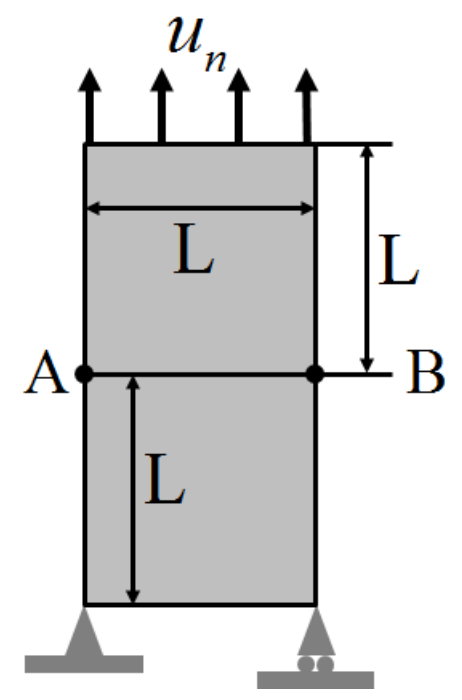

Figure 2: Effect of $\lambda$ on damage evolution

\section{Numerical examples}

In this section, the performance of the proposed interface model is assessed. In Section 8.1, a pure Mode I separation case is considered and the effect of the penalty factor on the traction vs. crack opening curve and on the damage evolution law is shown. Convergence to the analytical solution in a pure Mode I delamination case is demonstrated in Section 8.2 through the double cantilever beam (DCB) test problem while convergence and accuracy for mixed-mode delamination are examined in Section 8.3. Dynamic response of the interface model is investigated in Section 8.4 in the context of a one-dimensional spall test similar to the one in Ref. [5, 7]. The problems in Sections 8.1-8.3 are considered to be static and an implicit solver with arc length control is used. The spall test in Section 8.4 is clearly dynamic and an explicit time integration scheme is employed. Because the penalty formulation is the common basis for the classical cohesive elements, we compare the results obtained through the DG model to the results of the corresponding penalty formulation in Eq. (66).

Furthermore, for ease of representation of the results, the following definition for the normal crack opening $\Delta_{n}$ is introduced

$$
\Delta_{n}=\llbracket \boldsymbol{u} \rrbracket \cdot \boldsymbol{n} .
$$

\subsection{Mode I separation}

An interface A-B is defined between two square $L \times L$ elements, with $L=1$, as shown in Fig 2. An isotropic material with Young's modulus $E=1$ and Poisson's ratio $v=0$ is assumed in the two elements, and the interface energy and strength are respectively equal to $\mathrm{G}_{\mathrm{I}}^{c}=\mathrm{G}_{\mathrm{II}}^{c}=10$ and $t_{\mathrm{I}}^{c}=t_{\mathrm{II}}^{c}=1$. A uniform vertical displacement, $u_{n}$, is applied to the top of the element. The displacement is increased from 0 to a value $u_{n}^{\max }$ that corresponds to complete failure of the interface. In Fig. 3a the traction is plotted against the displacement jump at the interface. For the DG solution, the component of the displacement jump normal to the interface is zero until the failure criterion is met and then it increases until complete separation, $\llbracket \boldsymbol{u} \rrbracket \cdot \boldsymbol{n}=u_{n}^{\max }$. Furthermore, increasing the penalty factor, $\lambda$, does not change the resulting traction-crack opening curve. Conversely, the traction-crack opening curves obtained from the penalty based formulation are clearly dependent on the value of the penalty factor and tend to approach the DG curve for large values of $\lambda$.

The influence of the penalty factor $\lambda$ on the damage evolution is shown in Fig. 3b. The crack onset predicted by the DG formulation is not affected by the penalty factor, whereas it is evident that initially steeper curves correspond to higher values of $\lambda$. When the penalty-based formulation is employed, the crack initiation depends on the choice of $\lambda$ and, as expected, for large values of $\lambda$ the predicted results approach the results obtained from the DG based formulation. 


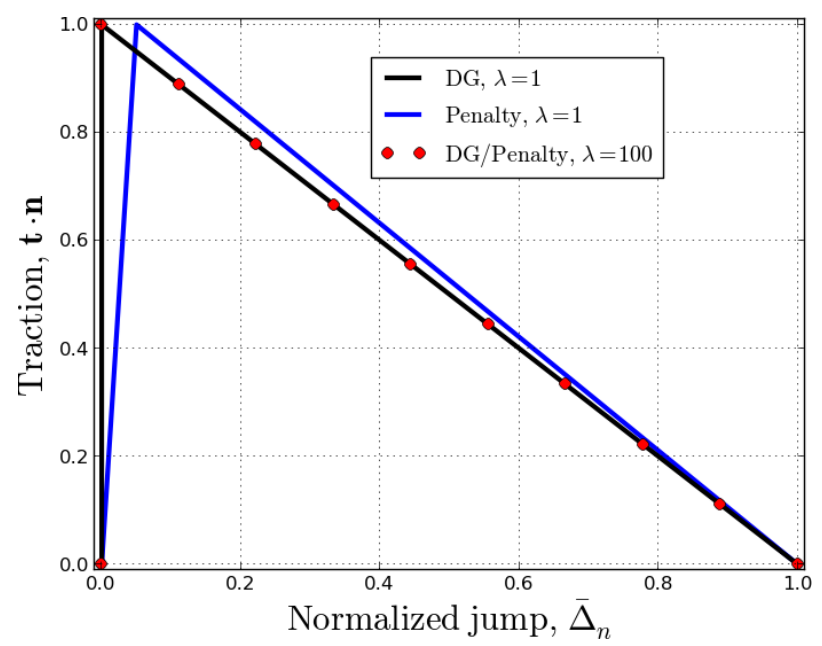

(a) Traction vs. normalized crack opening $\bar{\Delta}_{n}=\llbracket \boldsymbol{u} \rrbracket \cdot \boldsymbol{n} / u_{n}^{\max }$

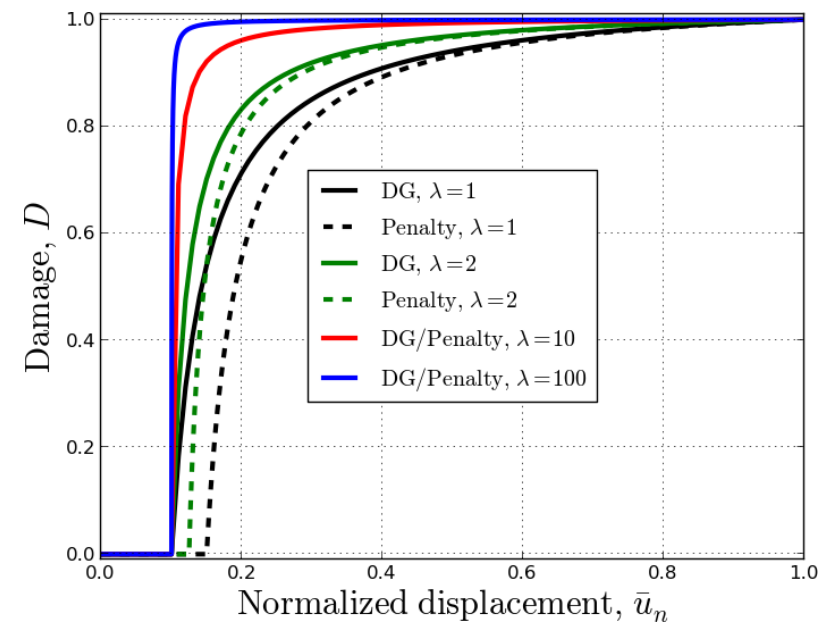

(b) Damage vs. normalized displacement $\bar{u}_{n}=u_{n} / u_{n}^{\max }$

Figure 3: Mode I delamination, resulting traction-separation law and influence of the scalar penalty $\lambda$.

\subsection{Double cantilever beam}

A beam of length $L=150 \mathrm{~mm}$, width $b=20 \mathrm{~mm}$ and thickness $h=3.96 \mathrm{~mm}$ is composed of two layers of equal thickness. Each layer is made of an orthotropic material with mechanical properties given in Table 1. The ends of the arms are subjected to equal and opposite loads, $P / 2$, as shown in Figure 4. This causes a pre-existing inter-laminar crack of initial length $a_{0}=55 \mathrm{~mm}$ to open and propagate toward the fixed end of the beam. Interfacial properties are given in Table 1.

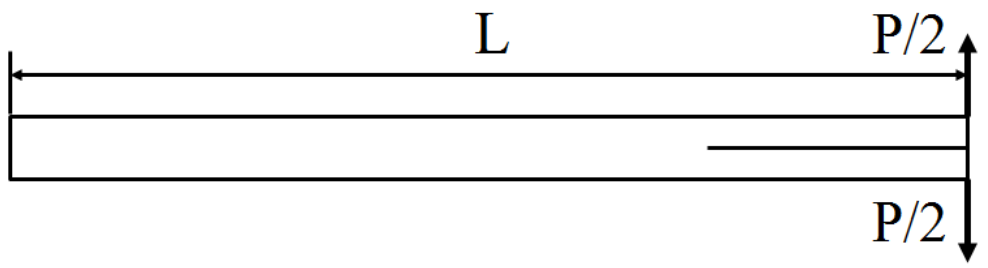

Figure 4: Double cantilever beam: geometry.

The load is increased until the component of the traction normal to the interface is $t_{n}^{*}=\boldsymbol{t}^{*} \cdot \boldsymbol{n}=t_{\mathrm{I}}^{c}$ at time $\tau=\tau_{c}$. Convergence of the solution and dependence of the crack propagation on the interface strength are investigated. Four levels of mesh refinement are considered, corresponding to element size $l_{e}=1.5 \mathrm{~mm}, l_{e}=1.0 \mathrm{~mm}, l_{e}=0.5 \mathrm{~mm}$, and $l_{e}=0.25 \mathrm{~mm}$. The mesh along the normal direction is not refined and one membrane element across each arm is employed. Plotting the load, $P$, against the crack opening displacement, in Figure 5a, shows that numerical results obtained using the current interface model converge to the analytical solution of Reeder et al. [36] as the mesh is refined. Figure $5 \mathrm{~b}$ shows the effect of the interface strength on the load-displacement response of the beam. Regardless of the interface strength, the crack propagation rate obtained from the DG formulation matches the analytical solution of Refs. [17, 30, 32]. As expected, reducing the interface strength value while keeping the interface energy constant causes damage to initiate earlier, which reduces somewhat the peak load.

\subsection{Mixed-mode bending test}

The ability of the present model to predict crack propagation under mixed mode fracture is demonstrated in the following model of a mixed-mode bending (MMB) test $[37,38]$. The configuration of the modeled specimen is the following: length, thickness and width are $2 L=150 \mathrm{~mm}, t=3.98 \mathrm{~mm}$ and $b=20 \mathrm{~mm}$ respectively. Moreover, the 
Table 1: Double cantilever beam: material and interface properties.

\begin{tabular}{llllllll}
\hline $\mathrm{E}_{11}$ & $\mathrm{E}_{22}=\mathrm{E}_{33}$ & $\mathrm{G}_{12}=\mathrm{G}_{13}$ & $\mathrm{G}_{23}$ & $v_{12}=v_{13}$ & $v_{23}$ & $\mathrm{G}_{\mathrm{I}}^{c}=\mathrm{G}_{\mathrm{II}}^{c}$ & $t_{\mathrm{I}}^{c}=t_{\mathrm{II}}^{c}$ \\
\hline $150.0 \mathrm{GPa}$ & $11.0 \mathrm{GPa}$ & $6.0 \mathrm{GPa}$ & $3.7 \mathrm{GPa}$ & 0.25 & 0.45 & $0.268 \mathrm{~N} / \mathrm{mm}$ & $45 \mathrm{MPa}$ \\
\hline
\end{tabular}

Table 2: Mixed-mode bending: material properties.

\begin{tabular}{llllll}
\hline $\mathrm{E}_{11}$ & $\mathrm{E}_{22}=\mathrm{E}_{33}$ & $\mathrm{G}_{12}=\mathrm{G}_{13}$ & $\mathrm{G}_{23}$ & $v_{12}=v_{13}$ & $v_{23}$ \\
\hline $120.0 \mathrm{GPa}$ & $10.5 \mathrm{GPa}$ & $5.25 \mathrm{GPa}$ & $3.48 \mathrm{GPa}$ & 0.3 & 0.45 \\
\hline
\end{tabular}

Table 3: Mixed-mode bending: interface properties.

\begin{tabular}{lllll}
\hline $\mathrm{G}_{\mathrm{I}}^{c}$ & $\mathrm{G}_{\mathrm{II}}^{c}$ & $t_{\mathrm{I}}^{c}$ & $t_{\mathrm{II}}^{c}$ & $\eta$ \\
\hline $0.260 \mathrm{~N} / \mathrm{mm}$ & $1.002 \mathrm{~N} / \mathrm{mm}$ & $30 \mathrm{MPa}$ & $60 \mathrm{MPa}$ & 2 \\
\hline
\end{tabular}

Table 4: Mixed-mode bending: number of iterations necessary to complete 100 increments

\begin{tabular}{llll}
\hline & $\lambda=1$ & $\lambda=10$ & $\lambda=100$ \\
\hline DG, non-symmetric & 592 & 647 & 778 \\
DG, symmetric & 605 & 648 & 784 \\
Penalty, non-symmetric & 613 & 641 & 756 \\
\hline
\end{tabular}

Table 5: Maximum stable time increment, [ns]

\begin{tabular}{lll}
\hline$\lambda$ & DG & Penalty \\
\hline $1 \times 10^{-5}$ & 10.00 & 10.00 \\
0.1 & 10.00 & 9.535 \\
1 & 9.429 & 7.071 \\
10 & 3.161 & 3.015 \\
100 & 1.000 & 0.995 \\
1000 & 0.316 & 0.316 \\
\hline
\end{tabular}




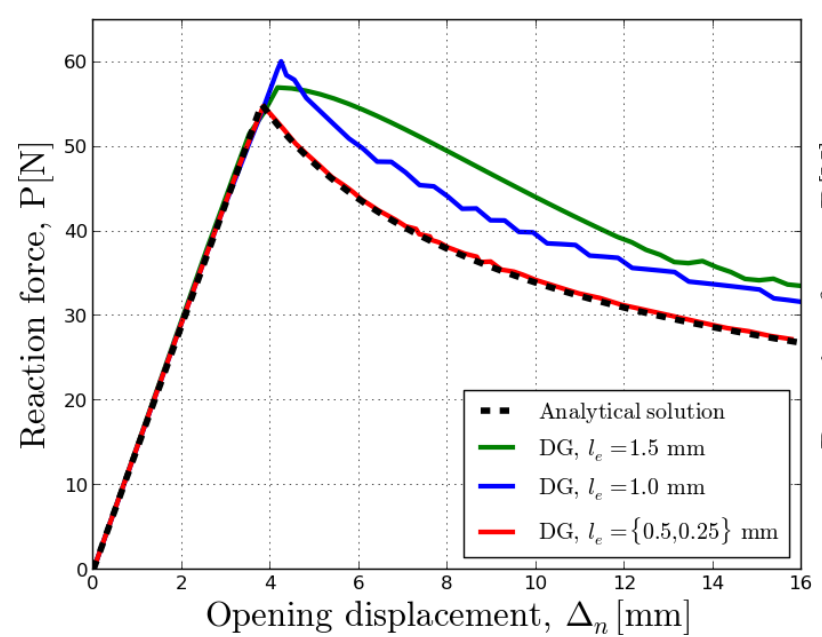

(a) Effect of mesh size

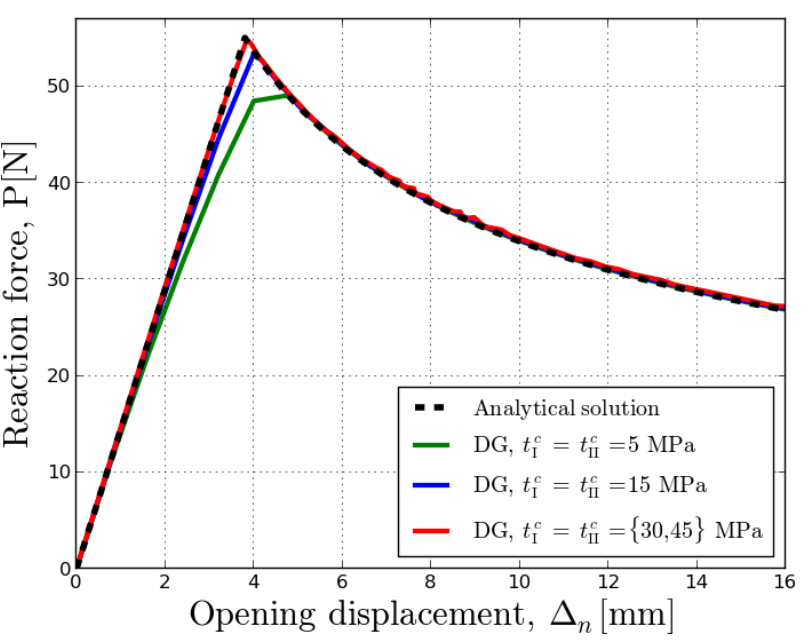

(b) Effect of interface strength, $l_{e}=0.25 \mathrm{~mm}$

Figure 5: Double cantilever beam, $\lambda=1$ : load vs. crack opening.

specimen has an initial delamination of length $a_{0}=35 \mathrm{~mm}$ and the load is applied through a rigid lever of length $L+c$, see Figure 6. Results obtained with the proposed interface model are compared against the analytical solution given in Ref. [30] for the three mode ratios considered: $\mathrm{B}=20 \%, \mathrm{~B}=50 \%$ and $\mathrm{B}=80 \%$ corresponding to $c=149.05 \mathrm{~mm}$, $c=63.18 \mathrm{~mm}$ and $c=41.06 \mathrm{~mm}$ respectively. The specimen's material properties are given in Table 2, whereas the interface properties are given in Table 3. In Figure 7, a mode ratio of 50\% is considered.

The convergence of the solution to the reference solution for three different meshes is shown in Figure 7a. The results in Figure $7 \mathrm{~b}$ indicate that changing the ratio of the interface strengths leads to negligible changes in the loaddeflection curves. The interface model provides accurate results also when different mode mixities are considered, $\mathrm{B}=20 \%$ in Figure $8 \mathrm{a}$ and $\mathrm{B}=80 \%$ in Figure 8b. Moreover, increasing the value of the penalty factor $\lambda$ does not affect the amount of energy dissipated during the delamination process and hence does not influence the loaddeflection curve, Figure 8 b.

The computational efficiency of the interface model has been investigated and the results are shown in Table 4. A mode ratio of $50 \%$ is considered and a fine mesh is used $\left(l_{e}=1 / 6 \mathrm{~mm}\right)$. The lever displacement is applied in 100 equal increments. Three formulation are considered: I) the non-symmetric DG that employs the $\mathbf{K}_{I}^{\Gamma}$ tangent matrix, II) the symmetric DG that uses the symmetricized tangent $\mathbf{K}_{I}^{\Gamma S}$ and III) non-symmetric penalty formulation (in Section 6) with the non-symmetric consistent tangent $\mathbf{K}_{I}^{\Gamma}$. The load-deflection curves obtained from the three formulations are identical (red curve in Figure 7a). The differences in the required number of iterations for the three methods are insignificant. Furthermore, increasing the value of the penalty factor $\lambda$ increases somewhat the number of iterations required to complete the analysis.

\subsection{Spall problem}

A spall impact problem is analyzed in order to compare the effects of the DG and penalty stiffness-based cohesive formulation on the wave propagation speeds inside a material containing interfaces, as well as the stable time increments required by both methods for explicit analysis.

The problem consists of a plate of thickness $\mathrm{L}_{1}=10 \mathrm{~mm}$ with initial velocity $v_{0}=100 \mathrm{~m} / \mathrm{s}$ colliding with a plate of thickness $\mathrm{L}_{2}=2 \mathrm{~L}_{1}$ initially at rest (Figure 9). The two plates are made of the same material with Young's modulus $E=2 \times 10^{5} \mathrm{MPa}$, Poisson's ratio $v=0$ and density $\rho=2000 \mathrm{Kg} / \mathrm{m}^{3}$. Since the Poisson's ratio is null, the problem is one-dimensional, and the results do not depend on the lateral dimensions of the plate. The speed of the elastic wave in the two plates is identical, $c=0.1 \mathrm{~m} / \mu \mathrm{s}$, and hence the tension waves will meet in the middle of the impacted plate, $x=\mathrm{L}_{1} \mathrm{~mm}$, at time $\tau_{c}=3 \mu \mathrm{s}$. The impacted plate is discretized with three different meshes: I) 100 elements of length $l_{e}=100 \mu \mathrm{m}$, II) 1000 elements $\left(l_{e}=10 \mu \mathrm{m}\right)$ and III) 10000 elements $\left(l_{e}=1 \mu \mathrm{m}\right)$, with DG interfaces between 


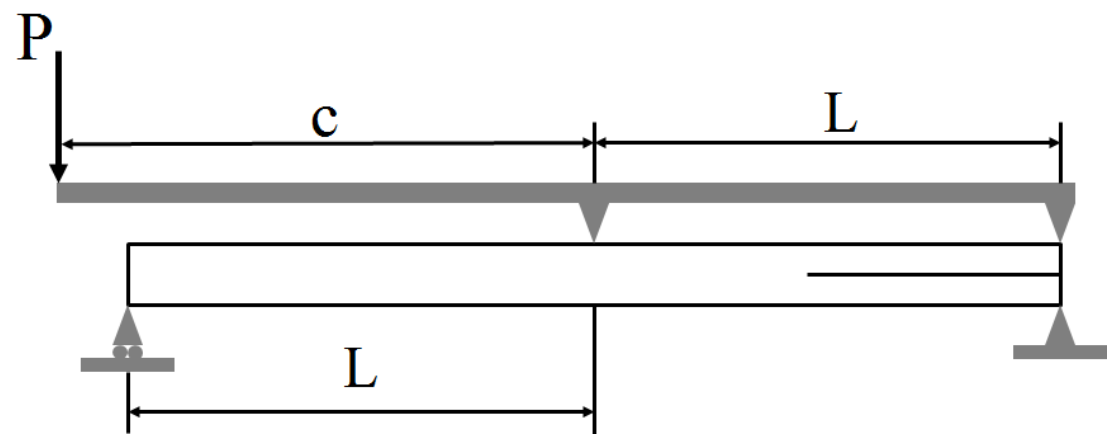

Figure 6: Mixed-mode bending: geometry.

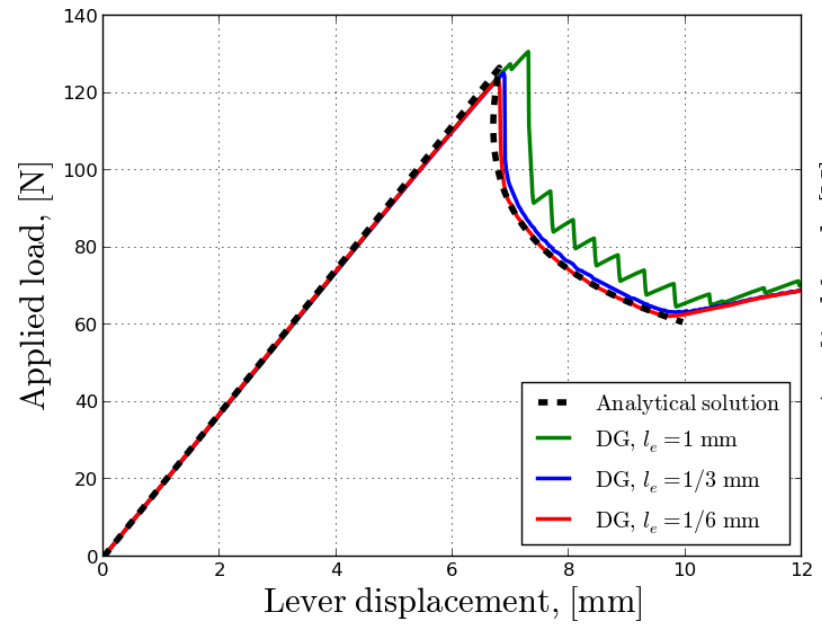

(a) Effect of mesh size

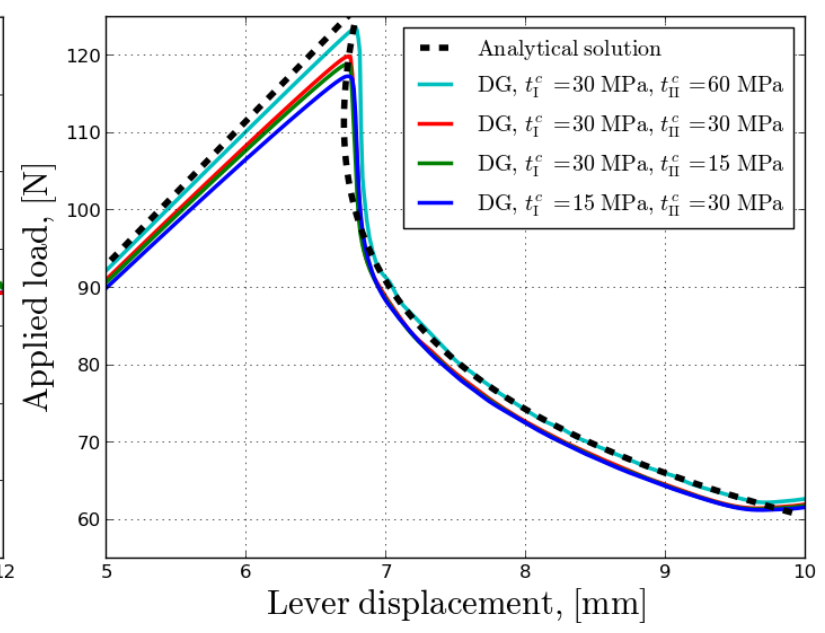

(b) Effect of interface strength, $l_{e}=1 / 6 \mathrm{~mm}$

Figure 7: MMB: load-displacement curve for $\mathrm{B}=50 \%, \lambda=1$.

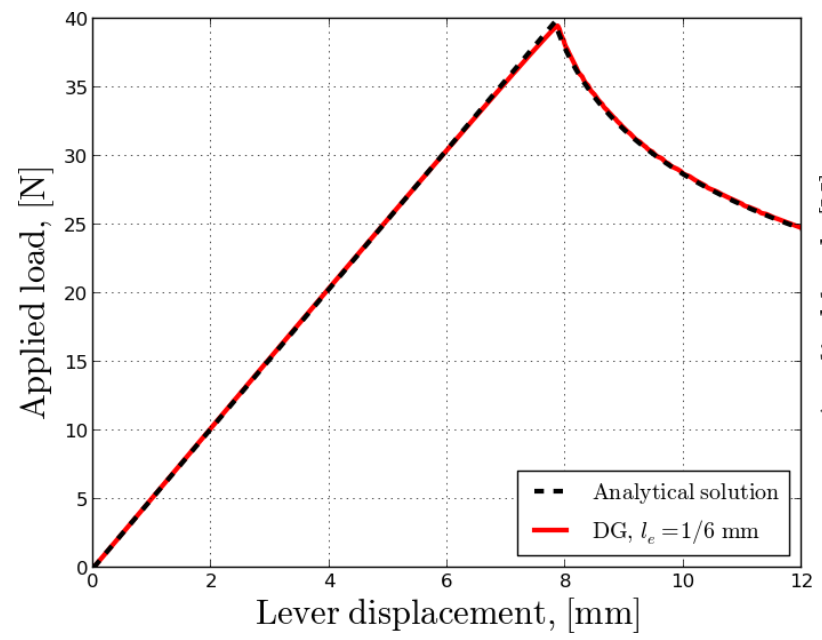

(a) $\mathrm{MMB}, \mathrm{B}=20 \%$

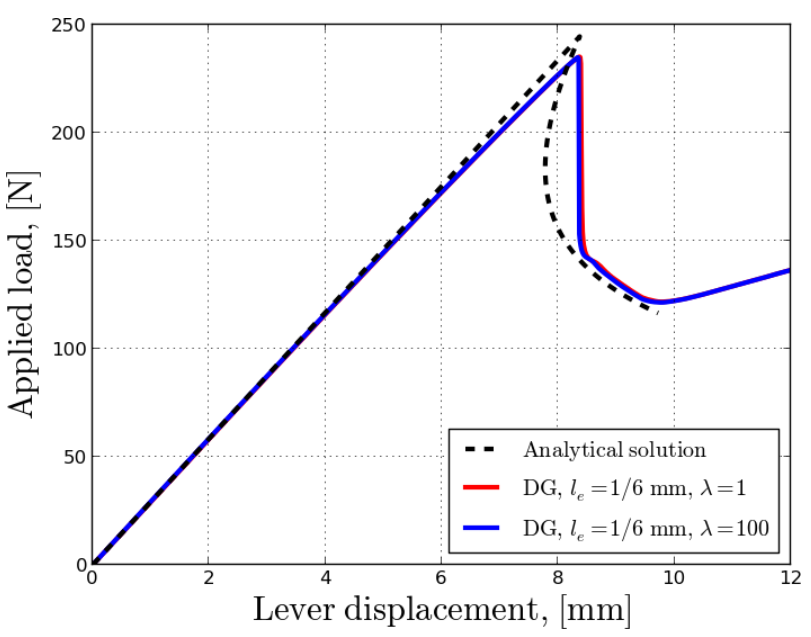

(b) MMB, B $=80 \%$

Figure 8: Dependence on the penalty factor, $\lambda$, of the pseudo-traction distribution near the crack tip. 


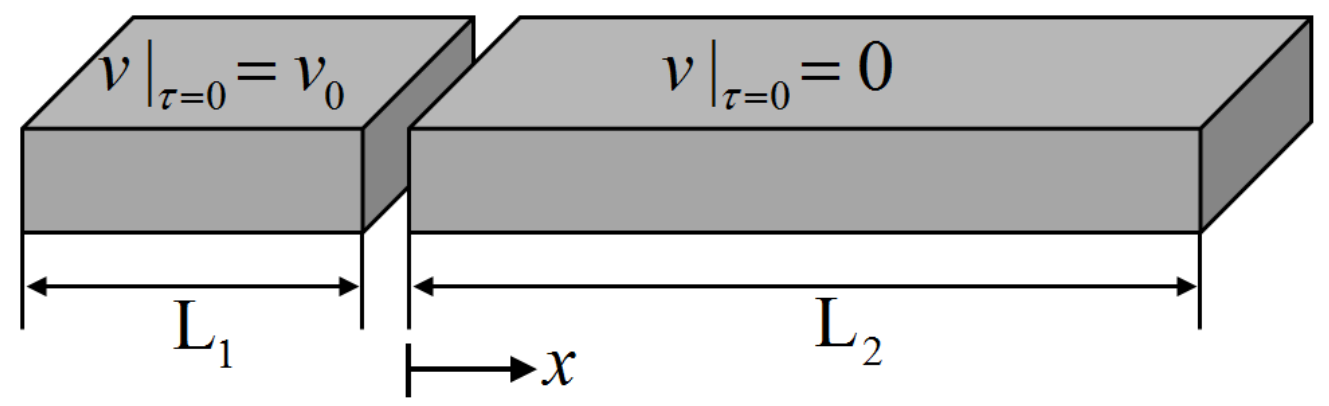

Figure 9: Spall problem geometry.

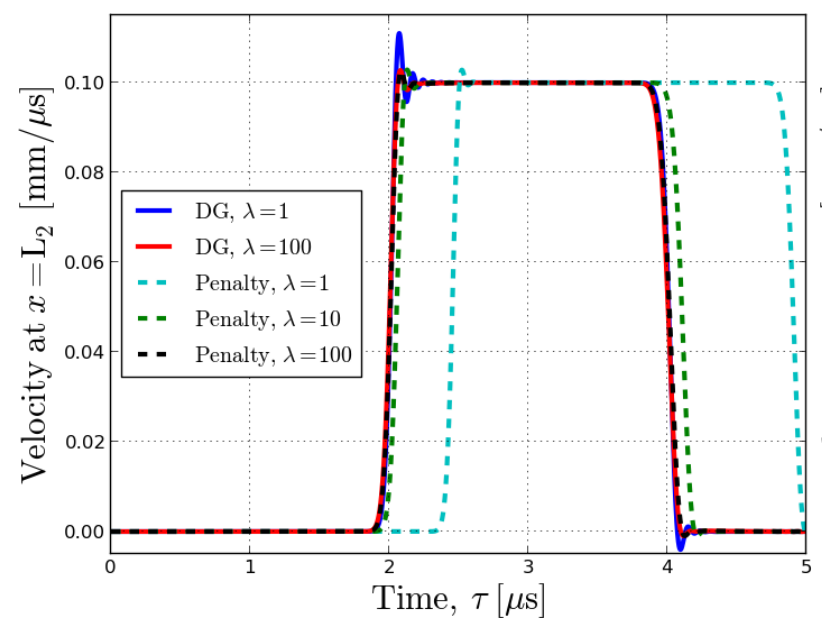

(a) Elastic interface behavior

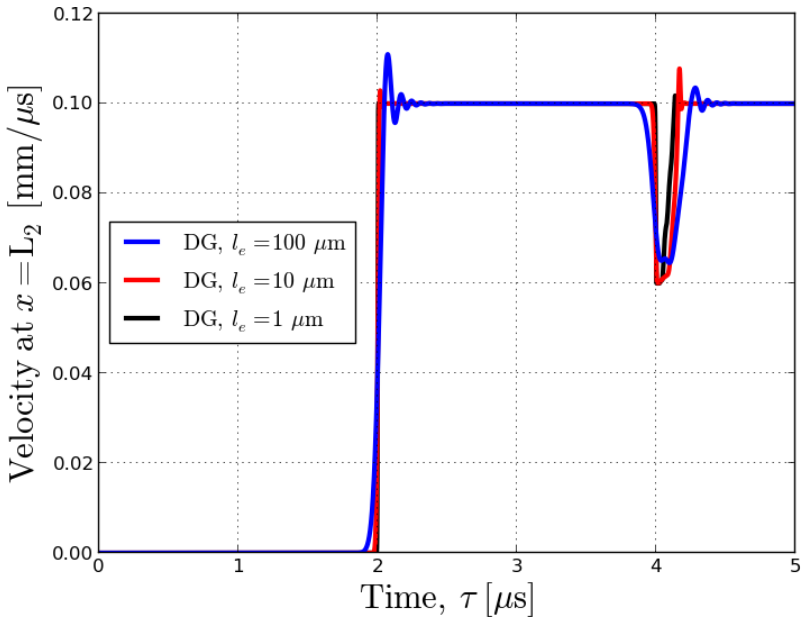

(b) Non-linear interface behavior, $\lambda=1$

Figure 10: Spall problem: velocity vs. time curves.

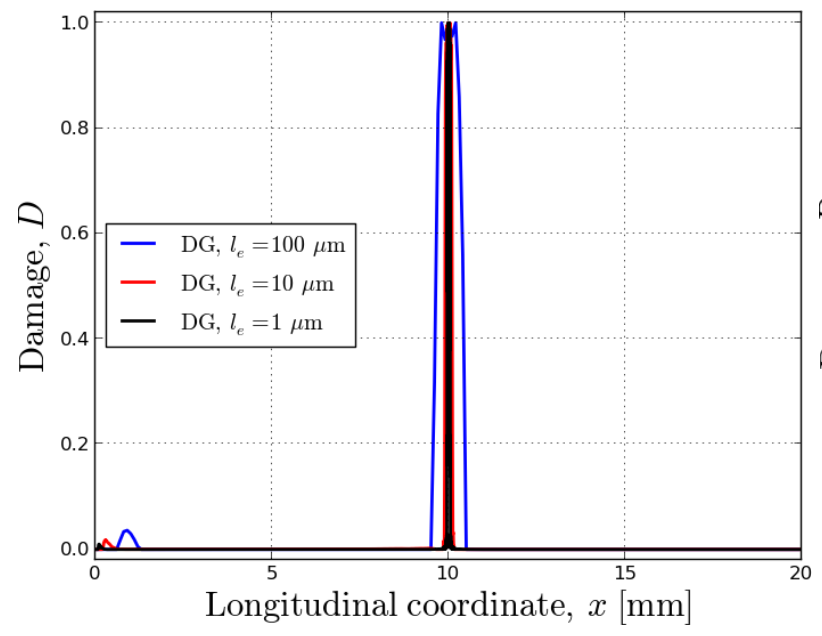

(a) Damage location on impacted plate

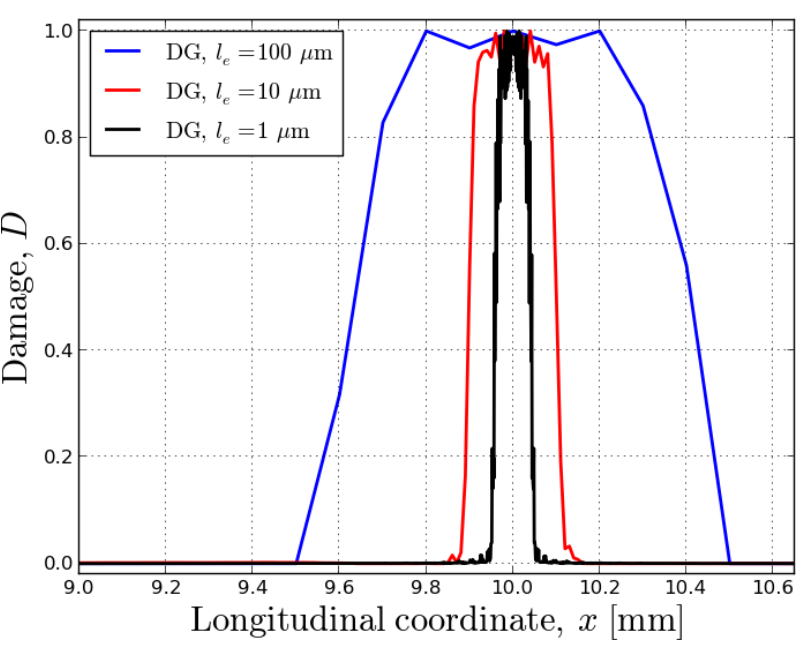

(b) Closeup of primary damage location

Figure 11: Spall problem: damage distribution, $\lambda=1$. 
all elements. All interfaces have fracture energy and fracture strength equal to $G=1 \mathrm{KJ} / \mathrm{m}^{2}$ and $t_{\mathrm{I}}^{c}=t_{\mathrm{II}}^{c}=400 \mathrm{MPa}$, respectively. Linear bulk viscosity is employed to reduce ringing (damp oscillations) [39].

In Figure 10, the velocity at the free surface $\left(x=\mathrm{L}_{2} \mathrm{~mm}\right)$ is plotted against time. The interface is assumed to behave elastically in Figure 10a, whereas in Figure 10b the DG formulation is coupled with the damage model. In the case of elastic interfaces, the results obtained with the penalty formulation appear to be highly dependent on the penalty factor. For low values of $\lambda$, the wave speed is highly underestimated, whereas for $\lambda=100$ the penalty and DG solution coincide, see Ref. [6]. Conversely, the DG formulation leads to results that are independent of the penalty factor. The highest frequency of the model is computed for several values of the penalty parameter $\lambda$, using mesh I, through a modal analysis. Subsequently, the maximum stable time increment is obtained from the highest frequecy $\Delta \tau_{\max }=\frac{2}{\omega_{\max }}$ for the symmetricized DG and penalty formulations, Table 5 . For a given $\lambda$, the difference between the maximum stable time increment for the DG and penalty formulations is negligible. Nonethess, the penalty formulation requires high penalty factors to be able to reproduce the correct elastic wave speed $(\lambda \approx 100)$ whereas the DG based formulation provides correct results for $\lambda=1$. Thus, it can be seen from Table 5 that the DG based formulation provides a maximum stable time increment that is one order of magnitude higher than that of the penalty formulation.

Due to the high influence of $\lambda$ on the elastic wave speed for the penalty formulation, the spall problem in presence of non-linear interface behavior is investigated with the DG formulation only. By comparing Figures 10a and 10b, the effect of the interface non-linearity on the time-velocity curve can be understood: the tension wave, originated during the damage process at $\tau \approx 3 \mu \mathrm{s}$, reaches the free surface at $\tau \approx 4 \mu$ s giving rise to the pullback signal [40]. In Figure $10 \mathrm{~b}$ it can be seen that the velocity measured at the free surface of the impacted plate converges when the mesh density is increased. Moreover, the velocity measured at $x=\mathrm{L}_{2} \mathrm{~mm}$ at $\tau \approx 4 \mu \mathrm{s}$ is $0.065 \mathrm{~mm} / \mu \mathrm{s}$ for mesh I and $0.060 \mathrm{~mm} / \mu$ s for meshes II and III.

The distribution of the damage, $D$, in the impacted plate is plotted against the longitudinal coordinate $x$ in Figure 11. The location of the primary fracture is computed correctly at $x=10 \mathrm{~mm}$, see Figure 11a, and damage is also predicted close to the impacted face of the plate at $\tau \approx 4 \mu \mathrm{s}$. Some mesh dependence is observed in Figure 11b: the damaged area becomes smaller when the mesh is refined while the number of damaged interfaces increases for finer meshes, reproducing the behavior observed in Ref. [7]. Noticeably the extent and magnitude of the damage close to the impacted face tend to diminish when the mesh is refined. We believe that the damage in proximity of the impacted face is due to numerical artifacts (ringing) which are also responsible for the lack of symmetry of the damage distribution at $x=\mathrm{L}_{1}$.

\section{Conclusions}

The present paper describes the formulation of a DG interface model for crack propagation analysis. The DG formulation is coupled with a damage variable that evolves in a thermodynamically consistent way. This allows the same DG energy functional to be used throughout the entire delamination process, obviating the need to replace it with a pure penalty formulation at the onset of fracture, as in hybrid DG-CZM methods.

It is noted that this thermodynamically consistent interface damage model can be used with classical penalty-based cohesive elements when implicit time stepping is employed. However, coupling this model with the DG method conveys significant advantages. Most importantly, in this case, the dissipated energy is independent of the penalty factor used to stabilize the DG formulation. In addition, when mixed-mode delamination is considered in the linear elastic fracture mechanics (LEFM) range, no correction of the interface strengths is necessary [30], since the crack propagation is governed by the interface fracture energy.

Although the consistent tangent matrix derived from the present formulation is non-symmetric, it has been shown that employing a symmetricized tangent matrix does not affect convergence characteristics. Further advantages of the present formulation over the classical cohesive-zone models have been demonstrated with the aid of a one-dimensional spallation problem. Numerical results reveal that the penalty term in the DG based formulation does not affect the wave speed, by contrast to classical penalty formulations which require large penalty factors for accuracy, thus placing stringent limits on the maximum size of the stable time increment in explicit analysis.

Future work directions include extending the current formulation to the large-deformation regime, generalizing it by including rate-dependent damage models, and treatment of fragmentation in homogeneous materials where interface locations are not know a priori. 


\section{Acknowledgments}

The work of D. Versino, H. M. Mourad and F. L. Addessio at Los Alamos National Laboratory was funded under the Joint DoD/DOE Munitions Technology Development Program (JMP). This support is gratefully acknowledged.

\section{References}

[1] E. F. Rybicki, M. F. Kanninen, A finite element calculation of stress intensity factors by a modified crack closure integral, Eng. Fract. Mech. 9 (1977) 931-938.

[2] D. S. Dugdale, Yielding of steel sheets containing slits, J. Mech. Phys. Solids 8 (1960) 100-104.

[3] G. Barenblatt, The mathematical theory of equilibrium cracks in brittle fracture, in: Advances in Applied Mechanics, volume 7, 1962, pp. $55-129$.

[4] J. R. Rice, A path independent integral and the approximate analysis of strain concentration by notches and cracks, J. Appl. Mech. 35 (1968) 379-386.

[5] R. Radovitzky, A. Seagraves, M. Tupek, L. Noels, A scalable 3D fracture and fragmentation algorithm based on a hybrid, discontinuous galerkin, cohesive element method, Comput. Methods Appl. Mech. Eng. 200 (2011) 326-344.

[6] H. D. Espinosa, P. D. Zavattieri, A grain level model for the study of failure initiation and evolution in polycrystalline brittle materials. Part I: Theory and numerical implementation, Mech. Mater. 35 (2003) 333-364.

[7] G. Camacho, M. Ortiz, Computational modelling of impact damage in brittle materials, Int. J. Solids Struct. 33 (1996) $2899-2938$.

[8] A. Pandolfi, M. Ortiz, Solid modeling aspects of three-dimensional fragmentation, Eng. with Comput. 14 (1998) $287-308$.

[9] J. Mergheim, E. Kuhl, P. Steinmann, A hybrid discontinuous Galerkin/interface method for the computational modelling of failure, Commun. Numer. Meth. Eng. 20 (2004) 511-519.

[10] L. Noels, R. Radovitzky, An explicit discontinuous Galerkin method for non-linear solid dynamics: Formulation, parallel implementation and scalability properties, Int. J. Numer. Meth. Eng. 74 (2008) 1393-1420.

[11] L. Wu, D. Tjahjanto, G. Becker, A. Makradi, A. Jérusalem, L. Noels, A micro-meso-model of intra-laminar fracture in fiber-reinforced composites based on a discontinuous galerkin/cohesive zone method, Eng. Fract. Mech. 104 (2013) 162-183.

[12] D. Versino, H. M. Mourad, T. O. Williams, A global-local discontinuous galerkin shell finite element for small-deformation analysis of multi-layered composites, Comput. Methods Appl. Mech. Eng. (2014).

[13] D. Versino, H. M. Mourad, T. O. Williams, F. L. Addessio, A global-local discontinuous galerkin finite element for finite-deformation analysis of multilayered shells, Comput. Methods Appl. Mech. Eng. 283 (2015) 1401-1424.

[14] K. Park, G. H. Paulino, J. R. Roesler, A unified potential-based cohesive model of mixed-mode fracture, J. Mech. Phys. Solids 57 (2009) 891-908.

[15] Y. Mi, M. A. Crisfield, G. A. O. Davies, H. B. Hellweg, Progressive delamination using interface elements, J. Compos. Mater. 32 (1998) $1246-1272$.

[16] M. Ortiz, A. Pandolfi, Finite-deformation irreversible cohesive elements for three-dimensional crack-propagation analysis, Int. J. Numer. Meth. Eng. 44 (1999) 1267-1282.

[17] G. Alfano, M. A. Crisfield, Finite element interface models for the delamination analysis of laminated composites: mechanical and computational issues, Int. J. Numer. Meth. Eng. 50 (2001) 1701-1736.

[18] P. Camanho, C. G. Dávila, Mixed-Mode Decohesion Finite Elements for the Simulation of Delamination in Composite Materials, Technical Report TM-2002-211737, NASA, 2002.

[19] A. Turon, P. Camanho, J. Costa, C. Dávila, A damage model for the simulation of delamination in advanced composites under variable-mode loading, Mech. Mater. 38 (2006) 1072-1089.

[20] J. Douglas Jr., T. Dupont, Interior penalty procedures for elliptic and parabolic Galerkin methods, in: R. Glowinski, J. L. Lions (Eds.), Computing methods in applied sciences, volume 58 of Lecture Notes in Physics, Springer, Berlin, 1976, pp. $207-216$.

[21] D. N. Arnold, An interior penalty finite element method with discontinuous elements, SIAM J. Numer. Anal. 19 (1982) $742-760$.

[22] D. Arnold, F. Brezzi, B. Cockburn, D. Marini, Discontinuous Galerkin methods for elliptic problems, in: B. Cockburn, G. E. Karniadakis, C.-W. Shu (Eds.), Discontinuous Galerkin Methods, volume 11 of Lecture Notes in Computational Science and Engineering, Springer, Berlin, 2000, pp. 207-216.

[23] W. Knausch, On the steady propagation of a crack in a viscoelastic sheet: Experiments and analysis, in: H. H. Kausch, J. A. Hassell, R. I. Jaffee (Eds.), Deformation and Fracture of High Polymers, 1973, pp. 501-541.

[24] J. R. Rice, The mechanics of quasi-static crack growth, in: Proc. 8th U.S. Nat. Cong. Appl. Mech., 1979, pp. 191-215.

[25] V. K. Goyal, E. R. Johnson, C. G. Dávila, Irreversible constitutive law for modeling the delamination process using interfacial surface discontinuities, Compos. Struct. 65 (2004) 289-305.

[26] M. L. Benzeggagh, M. Kenane, Measurement of mixed-mode delamination fracture toughness of unidirectional glass/epoxy composites with mixed-mode bending apparatus, Compos. Sci. Technol. 56 (1996) 439-449.

[27] D. N. Arnold, F. Brezzi, B. Cockburn, L. D. Marini, Unified analysis of discontinuous Galerkin methods for elliptic problems, SIAM J. Numer. Anal. 39 (2002) 1749-1779.

[28] J. Simo, J. Ju, Strain- and stress-based continuum damage models-I. Formulation, Int. J. Solids Struct. 23 (1987) 821-840.

[29] J. R. Reeder, An Evaluation of Mixed-Mode Delamination Failure Criteria, Technical Report, NASA, 1992.

[30] A. Turon, P. Camanho, J. Costa, J. Renart, Accurate simulation of delamination growth under mixed-mode loading using cohesive elements: Definition of interlaminar strengths and elastic stiffness, Compos. Struct. 92 (2010) 1857-1864.

[31] T. J. R. Hughes, The finite element method: linear static and dynamic finite element analysis, Dover Publications, Mineola, NY, 2000.

[32] A. Turon, C. Dávila, P. Camanho, J. Costa, An engineering solution for mesh size effects in the simulation of delamination using cohesive zone models, Eng. Frac. Mech. 74 (2007) 1665-1682. 
[33] ABAQUS 6.12, Abaqus Analysis User's Manual, Dassault Systèmes Simulia Corp., Providence, RI, USA, 2012.

[34] C. G. Dávila, C. A. Rose, E. V. Iarve, Modeling fracture and complex crack networks in laminated composites, in: V. Mantič (Ed.), Mathematical Methods and Models in Composites, volume 5 of Computational and experimental methods in structures, Imperial College Press, London, 2014, pp. 297-347.

[35] B. F. Sørensen, S. Goutianos, T. K. Jacobsen, Strength scaling of adhesive joints in polymer-matrix composites, Int. J. Solids Struct. 46 (2009) 741-761.

[36] J. R. Reeder, K. Demarco, K. S. Whitley, The use of doubler reinforcement in delamination toughness testing, Composites Part A 35 (2004) $1337-1344$.

[37] J. R. Reeder, J. H. Crews Jr., Mixed-mode bending method for delamination testing, AIAA J. 28 (1990) 1270-1276.

[38] J. H. Crews Jr., J. R. Reeder, A Mixed-Mode Bending Apparatus for Delamination Testing, Technical Report, NASA, 1998.

[39] D. J. Benson, Computational methods in lagrangian and eulerian hydrocodes, Comput. Methods Appl. Mech. Eng. 99 (1992) 235-394.

[40] D. Grady, M. Kipp, The micromechanics of impact fracture of rock, Int. J. Rock Mech. Min. Sci. \& Geomech. Abstr. 16 (1979) $293-302$. 